This item was submitted to Loughborough's Research Repository by the author.

Items in Figshare are protected by copyright, with all rights reserved, unless otherwise indicated.

\title{
One- and two-page crossing numbers for some types of graphs
}

PLEASE CITE THE PUBLISHED VERSION

http://dx.doi.org/10.1080/00207160802524747

\section{PUBLISHER}

(c) Taylor \& Francis

\section{VERSION}

AM (Accepted Manuscript)

\section{LICENCE}

CC BY-NC-ND 4.0

\section{REPOSITORY RECORD}

He, Hongmei, A.M. Salagean, and Erkki Makinen. 2019. "One- and Two-page Crossing Numbers for Some Types of Graphs". figshare. https://hdl.handle.net/2134/14699. 
This item was submitted to Loughborough's Institutional Repository (https://dspace.lboro.ac.uk/) by the author and is made available under the following Creative Commons Licence conditions.

\section{creative
commons}

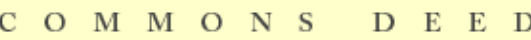

Attribution-NonCommercial-NoDerivs 2.5

You are free:

- to copy, distribute, display, and perform the work

Under the following conditions:

Attribution. You must attribute the work in the manner specified b the author or licensor.

Noncommercial. You may not use this work for commercial purposes.

No Derivative Works. You may not alter, transform, or build upon this work.

- For any reuse or distribution, you must make clear to others the license terms of this work.

- Any of these conditions can be waived if you get permission from the copyright holder.

Your fair use and other rights are in no way affected by the above.

This is a human-readable summary of the Leqal Code (the full license).

\section{Disclaimer 만}

For the full text of this licence, please go to: http://creativecommons.org/licenses/by-nc-nd/2.5/ 


\title{
One- and two-page crossing numbers for some types of graphs
}

\author{
Hongmei $\mathrm{He}^{*}$ \\ Department of Engineering Mathematics,University of Bristol, UK \\ H.He@bristol.ac.uk \\ Ana Sălăgean \\ Department of Computer Science, Loughborough University, UK \\ A.M.Salagean@lboro.ac.uk \\ Erkki Mäkinen \\ Department of Computer Sciences,University of Tampere, Finland \\ em@cs.uta.fi
}

\begin{abstract}
The simplest graph drawing method is that of putting the vertices of a graph on a line (spine) and drawing the edges as half-circles on $k$ half planes (pages). Such drawings are called $k$-page book drawings and the minimal number of edge crossings in such a drawing is called the $k$-page crossing number. In a one-page book drawing, all edges are placed on one side of the spine, and in a two-page book drawing all edges are placed either above or below the spine. The one-page and two-page crossing numbers of a graph provide upper bounds for the standard planar crossing. In this paper, we derive the exact one-page crossing numbers for four-row meshes, present a new proof for the one-page crossing numbers of Halin graphs, and derive the exact two-page crossing numbers for circulant graphs $C_{n}\left(1,\left\lfloor\frac{n}{2}\right\rfloor\right)$. We also give explicit constructions of the optimal drawings for each kind of graphs.
\end{abstract}

\section{Introduction}

A drawing of a graph $G=(V, E)$ is a placement of the vertices into distinct points of the plane and a representation of edges $(u, v)$ by simple continuous curves connecting the corresponding points and not passing through any point corresponding to a vertex other than $u$ and $v$. A crossing is a common point of two edges of $G$. We assume that any two curves representing the edges of $G$ have at most one point in common and that two curves incident to the same vertex do not cross. The crossing number $\operatorname{cr}(G)$ of $G$ is defined as

\footnotetext{
*This research was done while the first author was at the Department of Computer Science, Loughborough University, supported by the EPSRC grant GR/S76694/01
} 
the minimum number of crossings over all possible drawings of $G$ in the plane with the above properties.

The crossing minimisation problem is of importance in the field of graph drawings. The aesthetical properties and readability of graphs (such as diagrams or maps) depend heavily on the number of crossings. Moreover, crossing minimisation is an important goal in Very Large Scale Integration (VLSI) circuit design [8] and quantum-dot cellular automata [10], for which a smaller crossing number means lower implementation costs.

The simplest graph drawing method is that of putting the vertices on a line (spine) and drawing the edges as half-circles on $k$ half planes (pages). Such drawings are called $k$-page book drawings and the minimal number of edge crossings in such a drawing is called the $k$-page crossing number [9] and denoted by $\nu_{k}(G)$. A $k$-page book drawing is equivalent to a $k$-colour outerplanar (circular) drawing, in which all vertices are placed on a circle and all edges are drawn as straight lines with $k$ colours. Only crossings of same-coloured straight-line edges are counted. An outerplanar drawing indicates a one-colour circular drawing, i.e., it is equivalent to a one-page book drawing.

In a one-page book drawing, all edges are placed on one side of the spine, and in a twopage book drawing, edges are placed either above or below the spine. To minimise the crossing number we have to find the optimal order for the vertices on the spine and, in the case of more than one page, we also have to optimally divide the edges into the different pages. Similarly, for an outerplanar drawing we need to find the optimal order of the vertices on the circle and, in the case of $k$-colour outerplanar drawing, we have to optimally colour the edges. Both the one-page and the two-page crossing minimisation problems are NP-complete [6,7]. The one-page and two-page book crossing numbers of a graph provide upper bounds for the standard planar crossing number of the graph.

In this paper, we derive the exact one-page crossing numbers for four-row meshes, present a new proof for the one-page crossing numbers of Halin graphs, and derive the exact twopage crossing numbers for circulant graphs $C_{n}\left(1,\left\lfloor\frac{n}{2}\right\rfloor\right)$. We also give explicit constructions for the optimal drawings for each kind of graphs.

\section{One-page crossing numbers for 4-row meshes}

Let $P_{m}$ be an $m$-vertex path. Let $P_{m} \times P_{n}$ denote the $m \times n$ mesh graph, i.e., the union of $m$ paths of type $P_{n}$ (called rows) and $n$ paths of type $P_{m}$ (called columns). Figure 1 (a)-(c) shows three four-row meshes.

Vertices on the border of the mesh (i.e., vertices of degrees 2 or 3) are called b-vertices; the remaining vertices are in-vertices. The edges on the border (i.e., edges connecting b-verices) are called b-edges; the remaining edges are in-edges.

It is conjectured in [5] that $\nu_{1}\left(P_{4} \times P_{n}\right)=4(n-2), n \geq 3$. We prove that the conjecture is true and, moreover, explicitly construct optimal drawings for four-row meshes. The proof is given in the following two sections. First we construct, in Section 2.1, outerplanar drawings for $P_{4} \times P_{n}$ with $4(n-2)$ crossings. Then in Section 2.2 , we prove that any outerplanar drawing for $P_{4} \times P_{n}$ has at least $4(n-2)$ crossings. Hence, the drawings presented in Section 2.1 are optimal. 


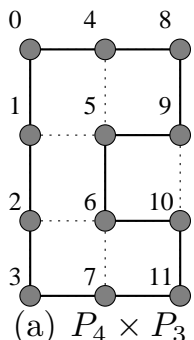

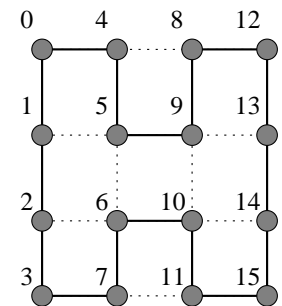

(b) $P_{4} \times P_{4}$

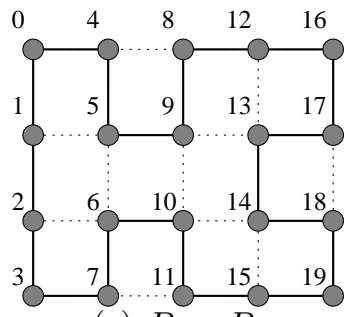

(c) $P_{4} \times P_{5}$

Figure 1: The conjectured optimal orders are indicated by the solid edges for $P_{4} \times P_{3}$, $P_{4} \times P_{4}$ and $P_{4} \times P_{5}$ respectively.

\section{$2.1 \quad$ Upper bound}

Figure 1 shows drawings for $P_{4} \times P_{3}, P_{4} \times P_{4}$, and $P_{4} \times P_{5}$, respectively. Outerplanar drawings of each mesh are obtained by placing the vertices on a circle in the order given by the Hamiltonian cycle drawn with solid lines. The numbers of crossings in these drawings are 4,8 , and 12 , respectively, i.e., $4(n-2)$. We verified the optimality of these drawings with a brute-force algorithm. For $n=2$, we can obviously draw $P_{4} \times P_{2}$ as an outerplanar drawing without any crossings.

Lemma 2.1. For a four-row mesh $P_{4} \times P_{n}, \nu_{1}\left(P_{4} \times P_{n}\right) \leq 4(n-2), n \geq 2$.

Proof. For the mesh $P_{4} \times P_{n}$, where $n$ is odd, the proposed optimal order is $0,4,5, \ldots, 4(n-$ $2), 4(n-1), 4(n-1)+1,4(n-2)+1,4(n-2)+2,4(n-1)+2,4(n-1)+3,4(n-2)+$ $3, \ldots, 3,2,1$ (Fig.2 (a)). For the mesh $P_{4} \times P_{n}$, where $n$ is even, the proposed optimal order is $0,4,5, \ldots, 4(n-1), 4(n-1)+1,4(n-1)+2,4(n-1)+3, \ldots, 3,2,1$ (Fig.3 (a)).

The proposed drawings can also be defined recursively. Given the drawing of $P_{4} \times P_{n}$ with $n$ odd, the optimal order of $P_{4} \times P_{n+1}$ is obtained by replacing the sequence $4(n-1), 4(n-$ $1)+1,4(n-2)+1,4(n-2)+2,4(n-1)+2,4(n-1)+3$ with $4(n-2)+1,4(n-1)+$ $1,4(n-1), 4 n, 4 n+1,4 n+2,4 n+3,4(n-1)+3,4(n-1)+2,4(n-2)+2($ Fig.2 (b)). For $n$ even, the drawing of $P_{4} \times P_{n+1}$ is obtained from the drawing of $P_{4} \times P_{n}$ by replacing the sequence $4(n-1)+1,4(n-1)+2$ with $4 n, 4 n+1,4(n-1)+1,4(n-1)+2,4 n+2,4 n+3$ (Fig.3 (a)).

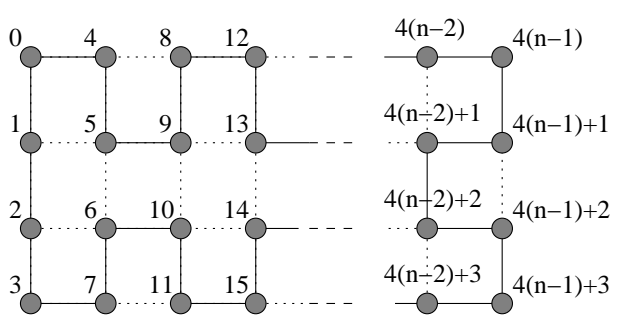

(a) $P_{4} \times P_{n}$

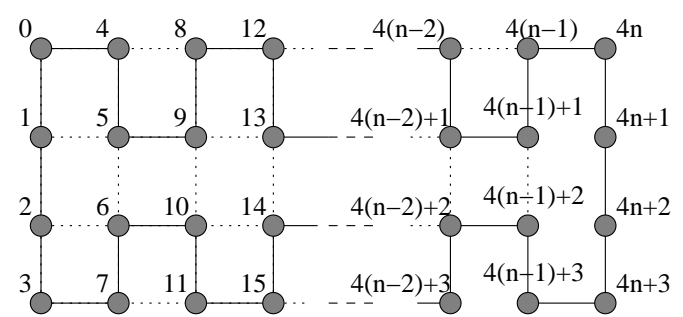

(b) $P_{4} \times P_{n+1}$

Figure 2: Optimal drawings for $P_{4} \times P_{n}$ and $P_{4} \times P_{n+1}, n$ is odd.

We now count the number of crossings (denoted by $c_{n}$ ) in the drawings constructed for $P_{4} \times P_{n}$. 


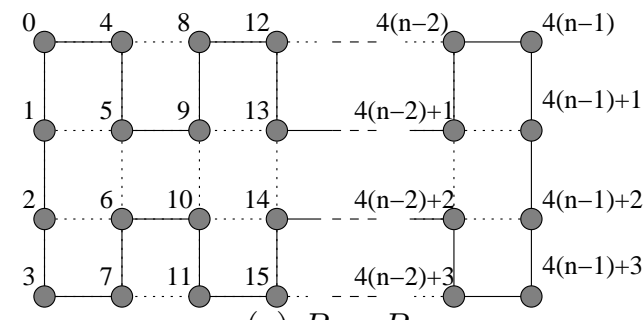

(a) $P_{4} \times P_{n}$

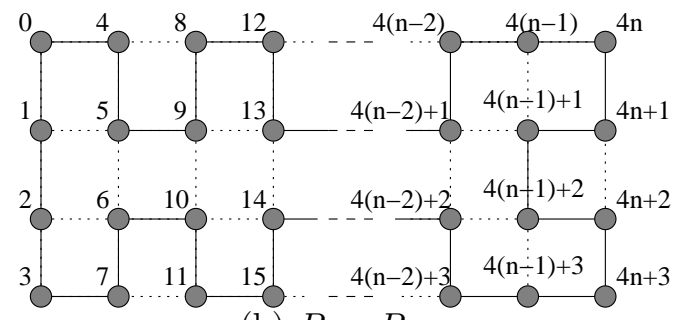

(b) $P_{4} \times P_{n+1}$

Figure 3: Optimal drawings for $P_{4} \times P_{n}$ and $P_{4} \times P_{n+1}, n$ is even.

For odd $n$, in Figure 2 (a), there is only one broken b-edge in the drawing of the last two columns, and in Figure 2 (b), there are two broken b-edges in the drawing of the last three columns. Each broken b-edge has 4 crossings, all of which involve in-edges.

Similarly, for $n$ even, in Figure 3 (a), there is no broken b-edge in the drawing of the last two columns, and in Figure 3 (b), there is one broken b-edge in the drawing of the last three columns. Again, each broken b-edge has 4 crossings, all of which involve in-edges.

Therefore, regardless of whether $n$ is odd or even, there is one more broken b-edge in the drawing of $P_{4} \times P_{n+1}$ than in the drawing of $P_{4} \times P_{n}$ and each broken b-edge produces exactly 4 crossings. Hence $c_{n+1}=c_{n}+4$. Combined with the initial condition $c_{3}=4$, we obtain that $c_{n}=4(n-2)$, i.e., the constructed drawings have exactly $4(n-2)$ crossings.

\subsection{Lower bound}

Lemma 2.2. For a four-row mesh $P_{4} \times P_{n}, \nu_{1}\left(P_{4} \times P_{n}\right) \geq 4(n-2), n \geq 2$.

Proof. Consider an arbitrary outerplanar drawing for $P_{4} \times P_{n}$. There are $2 n+4$ b-vertices and $2 n-4$ in-vertices. Each b-vertex is incident to two b-vertices and each b-vertex, except for the ones in the corners, is also incident to exactly one in-vertex. The b-edges form a cycle. The degree of an in-vertex is four. Each in-vertex, except for the two in column 2 and the other two in column $n-1$, is adjacent to exactly one b-vertex. The four in-vertices in columns 2 and $n-1$ are adjacent to exactly two b-vertices and these two b-vertices are not adjacent to each other.

We construct an outerplanar drawing as follows: First, all b-vertices are placed on a circle in the order they appear in the border of the mesh. This means that b-edges do not cross with each others. The in-vertices are then placed between the b-vertices.

When an in-vertex $v$ is inserted between two b-vertices $b_{1}$ and $b_{2}$, the b-edge $\left(b_{1}, b_{2}\right)$ crosses with either all four in-edges incident to $v$, if $v$ is adjacent to neither $b_{1}$ nor $b_{2}$ (see Figure $4(\mathrm{a})$ ), or three of the four in-edges incident to $v$, if $v$ is adjacent to either $b_{1}$ or $b_{2}$ (it cannot be adjacent to both $b_{1}$ and $b_{2}$ ) (see Figure $4(\mathrm{~b})$ ).

When two in-vertices $v_{1}$ and $v_{2}$ are inserted between two b-vertices $b_{1}$ and $b_{2}$, the b-edge $\left(b_{1}, b_{2}\right)$ crosses with either 2,3 or 4 of the edges incident to $v_{1}$ and either 2,3 or 4 of the edges incident to $v_{2}$. The case of two crossings with in-edges incident to $v_{1}$ and two crossings with in-edges incident to $v_{2}$ happens when the two in-vertices $v_{1}$ and $v_{2}$ are 
adjacent, and each of them is also adjacent to its neighbouring b-vertex on the circle (see Figure 5).

Assume next that we have an arbitrary number $t \geq 1$ of in-vertices, $v_{1}, v_{2}, \ldots, v_{t}$ (in this order) inserted between two b-vertices $b_{1}$ and $b_{2}$. Let $E_{0}$ be the set of edges with one endpoint in $V_{1}=\left\{v_{1}, v_{2}, \ldots, v_{t}\right\}$ and the other endpoint not in this set. Let $E_{1}$ be the set of edges with both endpoints in $V_{1}$. Since all vertices in $V_{1}$ have degree 4 , we have

$$
4 t=\left|E_{0}\right|+2\left|E_{1}\right| \text {. }
$$

All the edges in $E_{0}$ cross with the edge $\left(b_{1}, b_{2}\right)$, except for possibly two such edges, which connect vertices in $V_{1}$ to $b_{1}$ and to $b_{2}$. Hence, we have at least $\left|E_{0}\right|-2$ crossings so far.

At most $t-1$ of the edges are of the form $\left(v_{i}, v_{i+1}\right), 1 \leq i<t$, and they create no crossings. Any of the remaining $\left|E_{1}\right|-t+1$ edges is of the form $\left(v_{i}, v_{j}\right)$ with $j \geq i+2$. Consider first the case $j=i+2$. Out of the four edges incident with $v_{i+1}$, at least one is an edge incident to a b-vertex, so it is an edge in $E_{0}$, and out of the remaining three edges at most one is incident to $v_{i}$ or $v_{i+2}\left(v_{i+1}\right.$ cannot have edges incident to both $v_{i}$ and $v_{i+2}$ as there are no cycles of length 3 in the mesh). Hence, $\left(v_{i}, v_{j}\right)$ has at least three crossings in this case, one (at least) with an edge in $E_{0}$ and two (at least) with edges in $E_{1}$. The latter two crossings will be counted again when those edges are examined. So, to compensate for the double counting, we count the crossing between two edges in $E_{1}$ as half a crossing each time we encounter them. Therefore, $\left(v_{i}, v_{j}\right)$ has at least $1+1 / 2+1 / 2=2$ crossings. If $j>i+2$, there are at least two vertices between $v_{i}$ and $v_{j}$ and each of these has at least one edge incident to a b-vertex, i.e., an edge in $E_{0}$. This edge must cross with the edge $\left(v_{i}, v_{j}\right)$. So again, $\left(v_{i}, v_{j}\right)$ has at least two crossings. Altogether, at least $\left|E_{1}\right|-t+1$ of the edges in $E_{1}$ have crossings, and each of those has at least two crossings. There are at least $2\left|E_{1}\right|-2 t+2$ crossings involving two edges of $E_{1}$ or an edge of $E_{1}$ and an edge of $E_{0}$. Adding these to the $\left|E_{0}\right|-2$ crossings between $\left(b_{1}, b_{2}\right)$ and the edges of $E_{0}$, we obtain a total of $2\left|E_{1}\right|-2 t+2+\left|E_{0}\right|-2$ crossings. Using (1), this equals $2\left|E_{1}\right|-2 t+\left|E_{0}\right|=4 t-2 t=2 t$ crossings.

Regardless of the number of in-vertices inserted between two b-vertices $b_{1}$ and $b_{2}$, the number of crossings in the region of the plane enclosed by the edge $\left(b_{1}, b_{2}\right)$ and the segment of the circle delimited by $b_{1}$ and $b_{2}$ (and not containing any other b-vertices) is equal to at least twice the number of in-vertices placed between $b_{1}$ and $b_{2}$ on the circle. Since there are $2(n-2)$ in-vertices in total, we have at least $4(n-2)$ crossings.

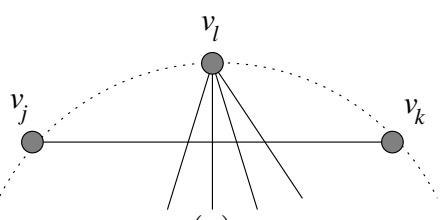

(a)

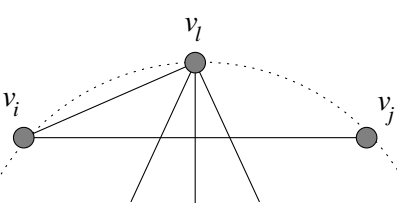

(b)

Figure 4: Two cases when an in-vertex is inserted between two adjacent b-vertices.

Next we examine what happens if the b-vertices are placed on the circle in an order different from that in the mesh border. In this case there are some crossings between b-edges. We prove that in addition to those, there still are at least $4(n-2)$ crossings involving an in-edge and a b-edge or two in-edges. 


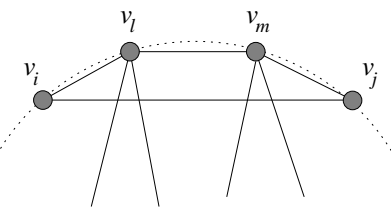

Figure 5: Two in-vertices are inserted between two adjacent b-vertices.

Consider two b-vertices $b_{1}$ and $b_{2}$ which are placed on the circle so that no other b-vertices are between them. Since the b-edges form a cycle, there are two different paths from $b_{1}$ to $b_{2}$ consisting of b-edges only. Assume that $b_{1}$ and $b_{2}$ are not adjacent. We break each b-edge into line segments delimited by the crossings with other b-edges. We then consider those line segments which form the shortest polygonal line connecting $b_{1}$ and $b_{2}$ (see the thick lines in Figure 6). Alternatively, this polygonal line can be defined as the polygonal line which, when taken together with the line segment $b_{1} b_{2}$, forms a convex polygon such that none of the b-edges has any point in the interior of this polygon. We refer to this polygonal line as the "shortest polygonal line" associated to $b_{1}$ and $b_{2}$. If $b_{1}$ and $b_{2}$ are adjacent, we define their shortest polygonal line to be the edge $\left(b_{1}, b_{2}\right)$. Note that the shortest polygonal lines of different pairs of b-vertices are all disjoint (except for possibly having endpoints of some of the segments in common).

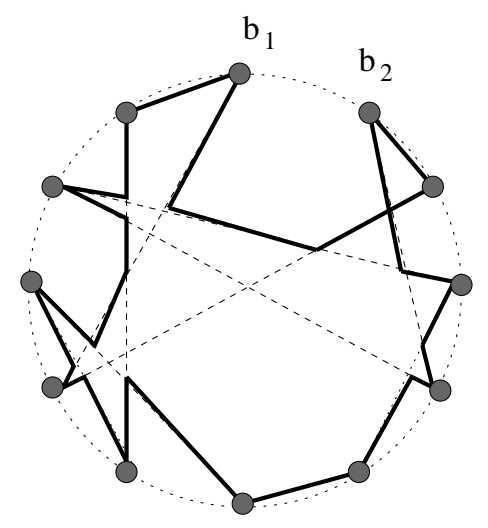

Figure 6: A random order of b-vertices on the circle for $P_{4} \times P_{4}$.

Next we insert the in-vertices between two b-vertices $b_{1}$ and $b_{2}$. The previous arguments hold when replacing all references to the edge $\left(b_{1}, b_{2}\right)$ by references to the shortest polygonal line of $b_{1}$ and $b_{2}$. As before, the number of crossings in the region of the plane enclosed by the shortest polygonal line of $b_{1}$ and $b_{2}$ and the segment of the circle delimited by $b_{1}$ and $b_{2}$ (and not containing any other b-vertices) is equal to at least twice the number of in-vertices placed between $b_{1}$ and $b_{2}$ on the circle. Since all these regions are pairwise disjoint no crossings are counted more than once. Furthermore, since there are $2(n-2)$ in-vertices in total, there are at least $4(n-2)$ crossings.

Therefore, we can conclude that the outerplanar crossing number of a 4-row mesh is not less than $4(n-2)$.

Lemmas 2.1 and 2.2 prove the following 
Theorem 2.3. For a four-row mesh $P_{4} \times P_{n}, \nu_{1}\left(P_{4} \times P_{n}\right)=4(n-2), n \geq 2$.

\section{One-page crossing numbers for Halin graphs}

A Halin graph $H$ is a planar graph $H=T \cup C$, where $T$ is a tree with no vertex of degree two and at least one vertex of degree three or more. $T$ in embedded in the plane and $C$ is a cycle connecting the leaves of $T$ in the cyclic order determined by the embedding of $T$. The edges in $T$ are called t-edges and the ones in $C$ are c-edges. A non-leaf vertex in a tree is called an in-vertex. A vertex is a central point of a graph if its eccentricity (the maximal distance to any other vertex) equals the graph radius. We assume a central point of a tree to be the root of the tree (vertex $v_{0}$ in Figure 7 and vertex 4 in Fig 8).
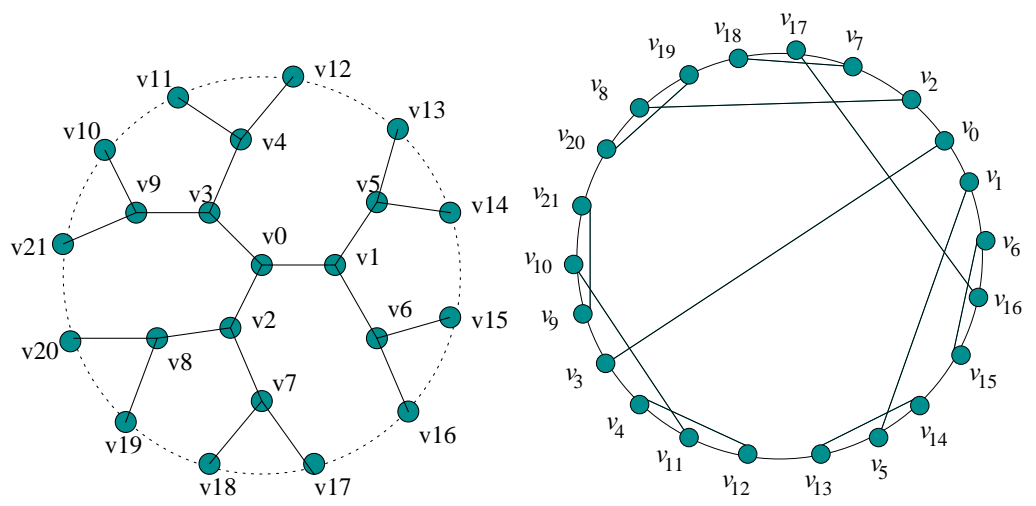

Figure 7: A Halin graph and its outerplanar drawing.

The one-page crossing number for Halin graphs with $\ell$ leaves is $\ell-2$ [2]. For example, the Halin graph in Figure 7, which has $\ell=12$ leaves, allows an outerplanar drawing with 10 crossings.

In this chapter we present a new proof for the one-page crossing number of Halin graphs, which is easier to follow than that given in [2]. Halin graphs are Hamiltonian [1]. Figure 8 shows that the solid edges form a Hamiltonian cycle. In the new version, we prove the upper bound of one-page crossing number for a Halin graph by providing an algorithm to construct a drawing based on a special Hamiltonian cycle for the Halin graph and calculating its crossing number. This algorithm for the construction of a drawing is totally different from the algorithm given in [2]. Moreover, the correctness of the algorithm in [2] was not included in the paper due to lack of space. We then prove the lower bound by giving a more detailed and rigorous version of the proof outlined in [2].

\subsection{Upper Bound}

Lemma 3.1. For a Halin graph $H$ with $\ell$ leaves, $\nu_{1}(H) \leq \ell-2$.

Proof. Given a Halin graph $H=T \cup C$ we construct an outerplanar drawing of $H$ with $\ell-2$ crossings. 


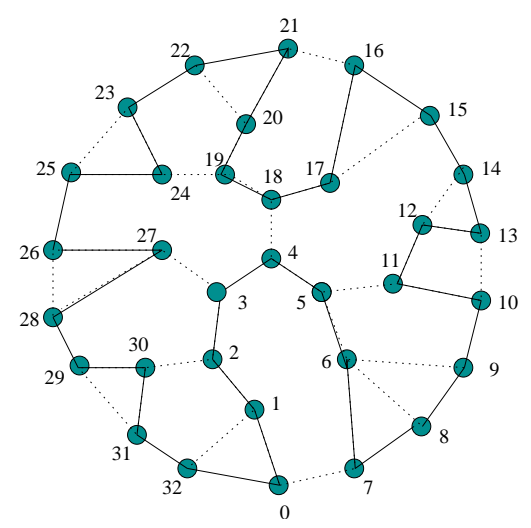

Figure 8: Optimal order vertices in a Halin graph.

Denote the leaves as $\left\{u_{0}, u_{1}, \ldots, u_{\ell-1}\right\}$ in the order they appear in the cycle $C$. We consider the indices of the leaves modulo $\ell$. For example, $u_{\ell}$ refers to $u_{0}$.

There is only one path between any pair of vertices in a tree. Hence, for each pair of leaves $u_{i}$ and $u_{i+1}$ which are neighbours on $C$, there is a unique path in $T$ connecting them. We denote this path by $P\left(u_{i}, u_{i+1}\right)=u_{i}, v_{i 1}, v_{i 2}, \ldots, v_{i n_{i}}, u_{i+1}$, where $n_{i}$ is the number of in-vertices on this path. Note that each vertex of the graph belongs to at least one such path. There is a subset of these paths which are pairwise disjoint and cover all the in-vertices and some of the leaves. Such a subset of paths can be determined and used to construct a Hamiltonian cycle by the following steps:

(1) Determine the paths $P\left(u_{0}, u_{1}\right), P\left(u_{1}, u_{2}\right), \ldots, P\left(u_{l-2}, u_{l-1}\right)$, and $P\left(u_{l-1}, u_{0}\right)$.

(2) Find the longest path $P\left(u_{i}, u_{i+1}\right)$.

(3) Remove all the vertices on the path $P\left(u_{i}, u_{i+1}\right)$ from $T$. This will separate the tree into several subtrees. The root of each subtree is the vertex that connects an invertex to the removed path. A subtree includes at least one in-vertex and two or more leaves unless the subtree is just a leaf.

(4) For all subtrees that are not a single leaf, repeat steps (2) and (3).

(5) For all independent paths found, replace the c-edge $\left(u_{i}, u_{i+1}\right)$ with $P\left(u_{i}, u_{i+1}\right)$ in $C$, i.e., insert the in-vertices $v_{i 1}, v_{i 2}, \ldots, v_{i n_{i}}$ between $u_{i}$ and $u_{i+1}$ on $C$ to form a Hamiltonian cycle (see Figure 9).

The outerplanar drawing is obtained by placing all vertices of $H$ on a circle in the order given by the Hamiltonian cycle constructed above. We will now compute the number of crossings in this drawing.

There are three possible types of intersecting edge pairs: pairs of t-edges, pairs of c-edges, and t-edges intersecting with c-edge. However, during the procedure above, the order of leaves on $C$ is not changed, so there are no crossings between c-edges in the drawing based on the constructed Hamiltonian cycle. 
According to the construction of the Hamiltonian cycle, each path comes from a separate subtree, the root of which is connected to one of the paths found previously. The inserted in-vertices are kept in the order they appear in the path. Furthermore, since the t-edges preserve their relative positions from the original planar embedding of the tree. So, no t-edge crosses with the constructed Hamiltonian cycle.

Each c-edge $\left(u_{i}, u_{i+1}\right)$ that has been replaced by the path $P\left(u_{i}, u_{i+1}\right)$ in the construction of the Hamiltonian cycle intersects some t-edges and all such t-edges have one end-vertex on the path $P\left(u_{i}, u_{i+1}\right)$. Out of the edges incident to a particular in-vertex on this path, two edges are on the path and the rest of the edges will have to cross the c-edge $\left(u_{i}, u_{i+1}\right)$ (see Figure 9).

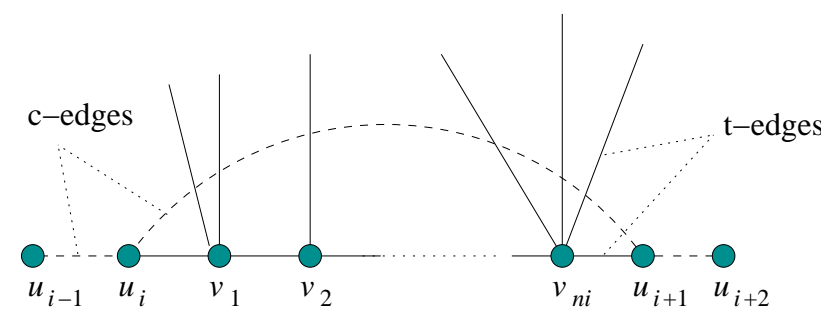

Figure 9: A path on the Hamilton cycle of Halin graph.

The c-edge $\left(u_{i}, u_{i+1}\right)$ therefore crosses with

$$
\sum_{j=1}^{n_{i}}\left(\operatorname{deg}\left(v_{i j}\right)-2\right)=\sum_{j=1}^{n_{i}} \operatorname{deg}\left(v_{i j}\right)-2 n_{i}
$$

edges, where $\operatorname{deg}(v)$ denotes the degree of a vertex $v$.

Let $i_{1}, i_{2}, \ldots, i_{k}$ be those values of the index $i$ for which $\left(u_{i}, u_{i+1}\right)$ has been replaced by the path $P\left(u_{i}, u_{i+1}\right)$ in the construction of the Hamiltonian cycle. The total number of crossings in the drawing is obtained by adding all the crossings of these c-edges:

$$
\sum_{t=1}^{k}\left(\sum_{j=1}^{n_{i_{t}}} \operatorname{deg}\left(v_{i_{t} j}\right)-2 n_{i}\right)=\sum_{t=1}^{k} \sum_{j=1}^{n_{i_{t}}} \operatorname{deg}\left(v_{i_{t} j}\right)-2 \sum_{t=1}^{k} n_{i_{t}} .
$$

Each in-vertex appears exactly once on the paths used in the Hamiltonian cycle. So the first term in (2) is the sum of degrees of all in-vertices in the tree of the Halin graph. A tree with $n$ vertices has $n-1$ edges, so the sum of all vertex degrees is $2(n-1)$. The sum of all in-vertex degrees is $2(n-1)-\ell$. The second term in $(2)$ is twice the number of in-vertices. There are $n-\ell$ in-vertices, so the number of crossings for the proposed drawing of the Halin graph is

$$
2(n-1)-\ell-2(n-\ell)=\ell-2 .
$$




\subsection{Lower Bound}

Lemma 3.2. For a Halin graph $H$ with $\ell$ leaves, $\nu_{1}(H) \geq \ell-2$.

Proof. The proof of the lower bound develops the ideas outlined in [2]. Given an outerplanar drawing of a Halin graph $H$, number the leaves of $H$ on the circle starting from the root in clockwise manner as $u_{0}, \ldots u_{\ell-1}$. The leaves of the tree of $H$ divide the circle into $\ell$ intervals of internal tree vertices. The intervals are either empty or contain some vertices of the tree of $H$. (See Figure 7.)

Notice that any edge between internal tree vertices of two different intervals crosses at least with two c-edges.

A t-edge incident with a leaf is called an l-edge; other t-edges are called internal t-edges. Now, consider the $\ell-2 l$-edges incident to a leaf other than $u_{0}$ and $u_{\ell-1}$. Let $\left(u_{i}, v\right)$ be such an edge, with $1 \leq i \leq \ell-2$ and $v$ an internal tree node. The vertex $v$ is in the interval between two successive leaves on the circle, $u_{j}$ and $u_{j+1}$. If $v$ is neither between $u_{i-1}$ and $u_{i}$ nor between $u_{i}$ and $u_{i+1}$, then the $l$-edge $\left(u_{i}, v\right)$ crosses with the c-edge $\left(u_{j}, u_{j+1}\right)$ (see Figure $10(\mathrm{a})$ ). Assume now that $v$ is in the interval between $u_{i}$ and $u_{i+1}$ (the case when it is between $u_{i-1}$ and $u_{i}$ is similar). In this case the l-edge has no crossings with c-edges. There is a unique path from $v$ to the root consisting only of $t$-edges. Consider the first edge $e$ in this path with one endpoint in the interval between $u_{i}$ and $u_{i+1}$ and the other endpoint in some other interval, say between $u_{k}$ and $u_{k+1}$ with $k \neq i$. The edge $e$ has at least two crossings, namely with the c-edges $\left(u_{i}, u_{i+1}\right)$ and $\left(u_{k}, u_{k+1}\right)$. Hence, each $l$-edge that has no crossings with c-edges is associated with an internal t-edge having at least two crossings with c-edges. The internal t-edge $e$ identified above can be associated to more than one $l$-edge. However, this is possible only if there exists an $l$-edge of the form $\left(u_{i+1}, v^{\prime}\right)$, with $v^{\prime}$ in the interval between $u_{i}$ and $u_{i+1}$ (possibly $v^{\prime}=v$ ) and $e$ appearing on the unique path from $v^{\prime}$ to the root. (see Figure $10(\mathrm{~b})$ ). However, the two crossings of $e$ will be counted at most twice. We can compensate the double counting by counting the two crossings of $e$ as one crossing only, each time the particular edge $e$ is associated to a $l$-edge. To conclude, each $l$-edge $\left(u_{i}, v\right)$ with $1 \leq i \leq \ell-2$ either has a crossing with a c-edge or there is a crossing crossing between a certain internal t-edge and a c-edge. Hence, there are at least $\ell-2$ crossings.

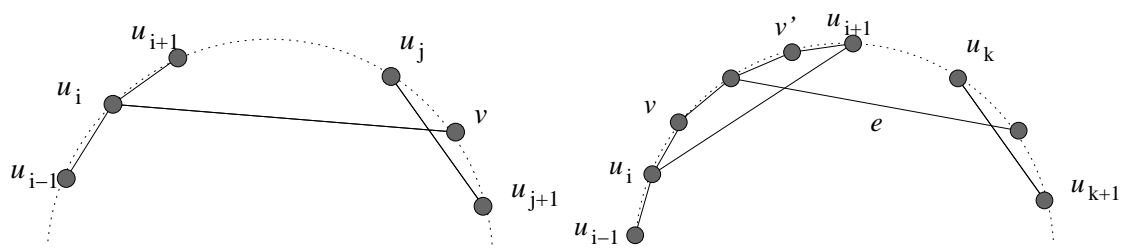

(a) An l-edge crosses with one c-edge (b) A t-edge crosses with two c-edges

Figure 10: Two cases in a outerplanar drawing for Halin graph.

We still have to consider the case where the leaves appear on the circle in an order different from the one given by the order on the cycle consisting of all the c-edges (in this case there are crossings involving two c-edges). Again, we number the leaves as $u_{0}, \ldots u_{\ell-1}$ in the order they appear on the circle, clockwise starting from the root. We apply the 
method used in Section 2.2. Namely, for each pair of leaves $u_{i}$ and $u_{i+1}$, we consider the shortest polygonal line connecting them and consisting of segments of c-edges. The proof then proceeds as above but any reference to an edge $\left(u_{i}, u_{i+1}\right)$ is replaced by a reference to the shortest polygonal line connecting $u_{i}$ and $u_{i+1}$. Therefore, there are at least $\ell-2$ crossings involving a t-edge and a c-edge, in addition to the crossings two c-edges.

Lemmas 3.1 and 3.2 prove the following

Theorem 3.3. For a Halin graph with $\ell$ leaves, $\nu_{1}=\ell-2$.

\section{Two-page crossing numbers for circulant graphs $C_{n}\left(1,\left\lfloor\frac{n}{2}\right\rfloor\right)$}

A circulant graph $C_{n}(1, k)$ has $n$ vertices $V=\{0,1,2, \ldots, n-1\}$, and edges connecting each vertex to the next one and to the $k$-th next one, i.e., $E=\{(i,(i+1) \bmod n),(i,(i+$ $k) \bmod n) \mid i \in V\}$ (see Figure 11 (a)). Circular graphs are regular Hamiltonian graphs.

In [4] we conjectured that the two-page crossing number for the special circulant graphs $C_{2 k}(1, k)$ is 1 , for $k \geq 3$. We now prove an even more general result.

Theorem 4.1. The two-page crossing number for a circulant graph $C_{n}\left(1,\left\lfloor\frac{n}{2}\right\rfloor\right), n \geq 6$, is $\nu_{2}\left(C_{n}\left(1,\left\lfloor\frac{n}{2}\right\rfloor\right)=1\right.$.

Proof. The two-page crossing number is an upper bound for the standard planar crossing number of a graph. The standard planar crossing number for $C_{n}\left(1,\left\lfloor\frac{n}{2}\right\rfloor\right)$ is known to be $1[11]$. Hence, $\nu_{2}\left(C_{n}\left(1,\left\lfloor\frac{n}{2}\right\rfloor\right) \geq 1\right.$.

Next we show that the two-page crossing number is at most 1, by giving an explicit 2colour outerplanar drawing with only one crossing. The drawing is guided by the heuristic of minimising the circular length of the edges. This heuristic has been used in [5] and, for the particular family of graphs $C_{n}\left(1,\left\lfloor\frac{n}{2}\right\rfloor\right)$, it gives the optimal solution.

We label the positions on the circle clockwise as $0,1, \ldots, n-1$, and the bijection $f: V \rightarrow$ $\{0,1, \ldots, n-1\}$ gives the position, for each vertex $v \in V$. The circular length of an edge $(u, v)$ in the drawing is defined as $\min \{|f(u)-f(v)|, n-|f(u)-f(v)|\}$. Intuitively, the length measures the number of positions we must move on the circle from the position of $u$ to the position of $v$ on the shorter route. The circular length of an outerplanar drawing of a graph is defined as the sum of the circular length of all the edges.

If we place the vertices of a circulant graph $C_{n}(1, k)$ in the order $0,1,2, \ldots, n-1$ on the circle (i.e., $f(i)=i$ ), we have $n$ edges of length 1 and either $k=n / 2$ edges of length $k$, if $n=2 k$, or $n$ edges of length $k$, otherwise. So, the circular length of such a graph drawing is $n+k^{2}$ or $n+n k$, respectively. For $C_{n}\left(1,\left\lfloor\frac{n}{2}\right\rfloor\right)$, the circular length is $k(k+2)$, for $n=2 k$, and $(k+1)(2 k+1)$, for $n=2 k+1$. Next, we try to obtain lower circular lengths by placing the vertices in a different order on the circle. The cases of even $n$ and odd $n$ are considered separately.

When $k$ is even, we rearrange the order of vertices as $1,1+k, 2+k, 2,3,(3+k) \bmod n,(4+$ $k) \bmod n, 4,5, \ldots, 0, k$. Note that this forms a Hamiltonian path rather than a cycle. 
(Intuitively, looking at the original drawing in which the vertices were placed in the order $0,1, \ldots, n-1$ we construct the Hamiltonian path by alternatively moving to the vertex opposite on the circle and then to the vertex immediately next on the circle in clockwise direction). For example in Figure 11 (b), the order of vertices is 1, 5, 6, 2, 3, 7, 0,4. The circular length becomes smaller, as there are $2 k-1$ edges with length 1 , two edges with length 2 , and $k-1$ edges with length 3 . Therefore, the circular length has been decreased from $k(k+2)$ to $2 k-1+2 \times 2+3(k-1)=5 k$.

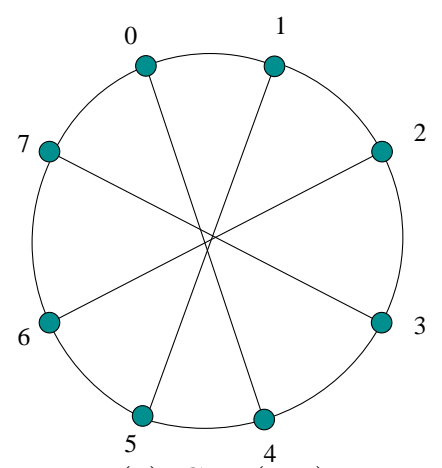

(a) $C_{2 \times 4}(1,4)$

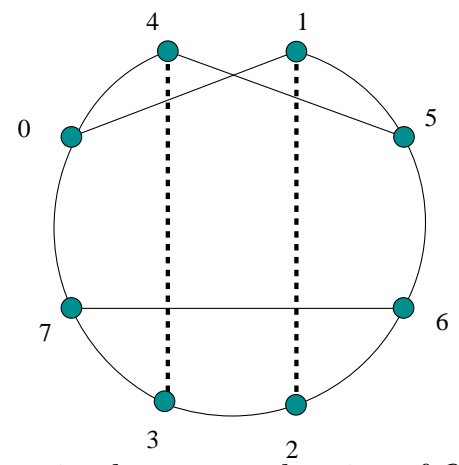

(b) The optimal two-page drawing of $C_{2 \times 4}(1,4)$

Figure 11: Circulant graph $C_{2 \times 4}(1,4)$ and its optimal two-page drawing.

When $k$ is odd, we rearrange the order of vertices as $1,1+k, 2+k, 2,3,(3+k) \bmod n,(4+$ $k) \bmod n, 4, \ldots, k, 0$. This forms a Hamiltonian cycle. Figure $12(\mathrm{~b})$ is the resulting drawing of $C_{2 \times 5}(1,5)$, and the order is $1,6,7,2,3,8,9,4,5,0$. There are $2 k$ edges of length 1 , and $k$ edges of length 3 . Therefore, the circular length is now $2 k+3 k=5 k$. The circular length has again reduced, except for the case $k=3$ where the length (and indeed the drawing) stays the same.

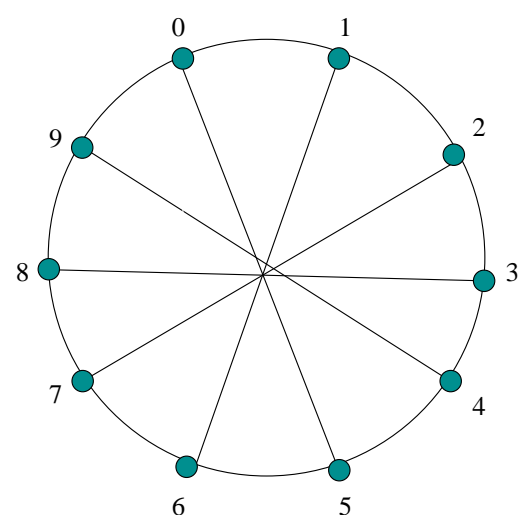

(a) $C_{2 \times 5}(1,5)$

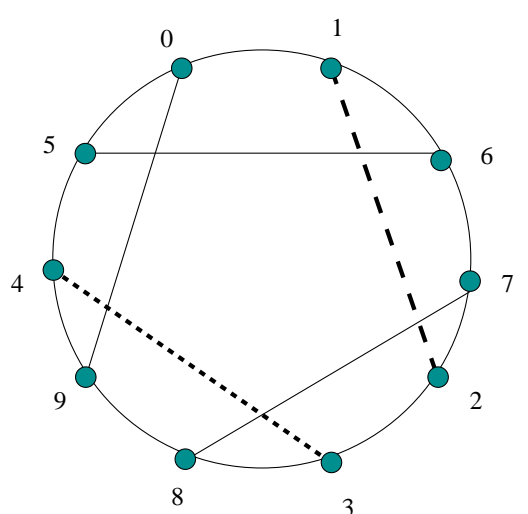

(b) The optimal two-page drawing of $C_{2 \times 5}(1,5)$

Figure 12: Circulant graph $C_{2 \times 5}(1,5)$ and its optimal two-page drawing.

Regardless of whether $k$ is odd or even, we clockwise pick those edges $(1,2),(3,4), \ldots$, $(t, t+1)$ with $t=k$, for odd $k$, and $t=k-1$, for even $k$. We colour these edges red, and all other edges blue. Red edges have length 3 and $f(1)<f(2)<f(3) \ldots<f(t)<f(t+1)$, so they do not intersect each other. 
For even $k$, the remaining edges of length higher than one are $(k, k+1),(k+2, k+$ $3), \ldots,(2 k-2,2 k-1),(0,1)$. Only the edges $(0,1)$ and $(k, k+1)$ cross. For odd $k$, the remaining edges of length higher than one are $(k, k+1),(k+2, k+3), \ldots,(2 k-1,0)$. Only the edges $(k, k+1)$ and $(2 k-1,0)$ cross. Therefore, we get a two-page drawing of a circulant graph $C_{2 k}(1, k)$ with only one crossing.

For $C_{2 k+1}(1, k)$ we rearrange the order of vertices on the circle as $0, k, 2 k \bmod n, 3 k \bmod$ $n \ldots,(n-1) k \bmod n$, which forms another Hamiltonian cycle. Figure $13(\mathrm{~b})$ is the resulting drawing of $C_{2 \times 4+1}(1,4)$, and the order is $0,4,8,3,7,2,6,1,5$. There are $2 k+1$ edges with length 1 , and $2 k+1$ edges with length 2 , therefore, the circular length is reduced from $(k+1)(2 k+1)$ to $2 k+1+2(2 k+1)=3(2 k+1)$.

We colour the edges, $(0,2 k \bmod n),(2 k \bmod n, 4 k \bmod n), \ldots,((n-3) k \bmod n,(n-1) k \bmod$ $n)$ red, and all other edges are coloured blue. Obviously, the $k$ red edges form a path and $f(0)<f(2 k \bmod n)<f(4 k \bmod n)<\ldots<f((n-1) k \bmod n)$. So, they will not create any crossing.

There are $k+1$ blue edges of length higher than one, namely $((n-1) k \bmod n, k),(k, 3 k \bmod$ $n),(3 k \bmod n, 5 k \bmod n), \ldots,((n-2) k \bmod n, 0)$, which again form a path. There are no crossings among the last $k$ edges of the path, as $f(k)<f(3 k \bmod n)<f(5 k \bmod n)<$ $\ldots<f((n-2) k \bmod n)$. However, the first edge on this path does cross with the last edge, namely $((n-1) k \bmod n, k)$ crosses with $((n-2) k \bmod n, 0)$. Therefore, there is only one crossing in the drawing constructed.

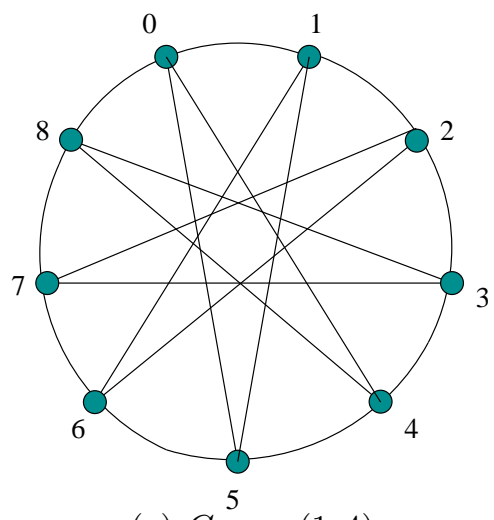

(a) $C_{2 \times 4+1}(1,4)$

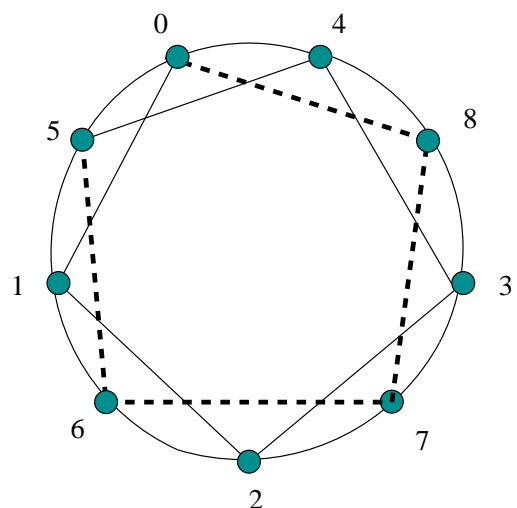

(b) The optimal two-page drawing of $C_{2 \times 4+1}(1,4)$

Figure 13: Circulant graph $C_{2 \times 4+1}(1,4)$ and its optimal two-page drawing.

\section{Conclusion}

We proved that the one-page crossing number for 4-row meshes $P_{4} \times P_{n}$ is $4(n-2)$. We also presented a new proof of the fact that the one-page crossing number of Halin graphs with $\ell$ leaves is $\ell-2$. Finally, we showed that the two-page crossing number for the special family of circulant graphs $C_{n}\left(1,\left\lfloor\frac{n}{2}\right\rfloor\right)$ equals one. We gave an explicit construction of the optimal drawing for each kind of graphs. 


\section{References}

[1] Cornuéjols, G., Naddef, D. and Pulleyblank, W. R., Halin Graphs and the travelling salesman problem. Math. Prog. 16 (1983), pp. 287-294.

[2] Fulek, R., He, H., Sýkora, O., and Vrťo, I., Outerplanar crossing numbers of 3-row meshes, Halin graphs and complete p-partite graphs, Proc. SOFSEM'05, LNCS 3381, Springer, (2005), pp. 376-379.

[3] Guy, R. K., Crossing number of graphs, in Y. Alavi, D. R. Lick and A. T. White, eds, Graph Theory and Applications: Proc. of the Conference at Western Michigan University, Kalamazoo, New York: Springer-Verlag, (1972), pp. 111-124.

[4] He, H., Sýkora, O., and Mäkinen, E., Genetic algorithms for the two-page book crossing number problem of graphs, Journal of Heuristics 13 (2007), pp. 77-93.

[5] He, H., Sýkora, O., Sălăgean, A. M., and Vrťo, I., Heuristic crossing minimisation algorithms for the two-page drawing problem, submitted for publication in $A C M$ Journal of Experimental Algrithmics.

[6] Masuda, S., Kashiwabara, T., Nakajima, K., and Fujisawa, T., On the NPcompleteness of a computer network layout problem, in: Proc. IEEE Intl. Symposium on Circuits and Systems 1987, IEEE Computer Society Press, Los Alamitos, (1987), pp. 292-295.

[7] Masuda, S., Nakajima, K., Kashiwabara, T., and Fujisawa, T., Crossing minimization in linear embeddings of graphs, IEEE Trans. Comput. 39 (1990), pp. 124-127.

[8] Mead, C., and Conway, L., Introduction to VLSI Systems. Addison-Wesley, 1979.

[9] Shahrokhi, F., Sýkora, O., Székely, L.A., and Vrt'o, I., The book crossing number of a graph, Journal of Graph Theory 21 (1996), pp. 413-424.

[10] Tougaw, P., and Lent, C., Logical devices implemented using quantum cellular automata, J. Appl. Phys. 75 (1994), pp. 1818-1825.

[11] Yang,Y., and Zhao, C., The crossing number of $C_{n}(1, k)$, in Proc. National Symposium on Software Technology held by Chinese Computer Institute, (2001), pp. 134-136. 